\title{
Profiling of transcripts and proteins modulated by the E7 oncogene in the lung tissue of E7-Tg mice by the omics approach
}

\author{
EUNJIN KIM ${ }^{1 *}$, JEONGWOO KANG ${ }^{1,3^{*}}$, MINCHUL CHO $^{1}$, SOJUNG LEE $^{1}$, EUNHEE SEO $^{1}$, HEESOOK CHOI $^{1}$, \\ YUMI KIM ${ }^{1}$, JUNGHEE KIM ${ }^{1}$, KUM YONG KANG ${ }^{2}$, KWANG PYO KIM ${ }^{2}$, JAEYONG HAN ${ }^{3}$, \\ YHUNYHONG SHEEN $^{4}$, YOUNG NA YUM ${ }^{5}$, SUE-NIE PARK ${ }^{5}$ and DO-YOUNG YOON ${ }^{1}$

\begin{abstract}
Departments of ${ }^{1}$ Bioscience and Biotechnology, and ${ }^{2}$ Molecular Biotechnology, Konkuk University, Hwayang-dong 1 , Seoul National University, Seoul 151-742; ${ }^{4}$ School of Pharmacy, Ewha Womans University, Seoul 120-750;

${ }^{5}$ Korea Food and Drug Administration, \#194 Tongil-ro, Eunpyung-gu, Seoul 122-704, Korea
\end{abstract} \\ Gwangjin-gu, Seoul 143-701; ${ }^{3}$ Laboratory of Animal Genetic Engineering, Department of Food and Animal Biotechnology,
}

Received August 18, 2008; Accepted November 10, 2008

DOI: $10.3892 / \mathrm{mmr} 00000073$

\begin{abstract}
The E6 and E7 oncoproteins of human papilloma virus (HPV) type 16 have been known to cooperatively induce the immortalization and transformation of primary keratinocytes. We established an E7 transgenic mouse model to screen HPV-related biomakers using the omics approach. The methods used to identify HPV-modulated factors were genomics analysis by microarray using the Affymetrix 430 2.0 array to screen E7-modulated genes, and proteomics analysis using nano-LC-ESI-MS/MS to screen E7-modulated proteins with the lung tissue of E7 transgenic mice. According to omics data, cyclin B1, cyclin E2, topoisomerase II $\alpha$, calnexin, activated leukocyte cell adhesion molecule CD166, actinin $\alpha 1$, diaphorase 1 , gelsolin, platelet glycoprotein, and annexin A2 and A4 were up-regulated in the E7-Tg mice, while proteoglycan 4 , sarcolipin, titin, vimentin, drep 1 , troponin and cofilin-1 were down-regulated. We further confirmed the significance of differences between the expression levels of the selected factors in E7-Tg and non-Tg mice by real-time PCR. Genes related to cancer cell adhesion, cell cycle and migration, proliferation and apoptosis, as well as to the intermediate filament network and to endoplasmic reticulum proteins, were selected. Taken together, the results
\end{abstract}

Correspondence to: Dr Do-Young Yoon, Department of Bioscience and Biotechnology, Konkuk University, Hwayang-dong 1, Gwangjin-gu, Seoul 143-701, Korea

E-mail: ydy4218@konkuk.ac.kr

Dr Sue Nie Park, Korea Food and Drug Administration, \#194 Tongil-Ro, Eunpyung-Gu, Seoul 122-704, Korea

E-mail: suenie@kfda.go.kr

*Contributed equally

Key words: proteomics, human papilloma virus, E7 oncogene, genomics, Tg mouse, cancer suggest that the E7 oncogene modulates the expression levels of cell cycle-related (cyclin B1, cyclin E2) and cell adhesionand migration-related (actinin $\alpha 1, \mathrm{CD} 166$ ) factors, which may play important roles in cellular transformation in cancer. In addition, the solubilization of the rigid intermediate filament network by specific proteolysis mediated via up-regulating gelsolin and down-regulating cofilin-1, as well as increased levels of endoplasmic reticulum protein calnexin with chaperone functions, might also be involved in E7-lung epithelial cells.

\section{Introduction}

Human papillomaviruses (HPVs) are small circular doublestranded DNA viruses that belong to the Papovaviridae family. HPV DNA has been identified in more than $95 \%$ of all cervical tumors, with types $16,18,31,33,45$ and 51 most frequently associated with genital malignancies (1). The E7 oncoproteins contain approximately 98 amino acids and incorporate three conserved regions, CR1, CR2 and CR3 (2). The CR1 domain consists of the amino terminus ( $\mathrm{NH}_{2}$-terminal) and is necessary for cellular transformation and retinoblastoma gene product (pRb) degradation, but does not contribute to $\mathrm{pRb}$ binding (3). $\mathrm{CR} 2$ comprises an $\mathrm{LxCxE}$ motif that mediates the binding of $\mathrm{E} 7$ to retinoblast $(\mathrm{Rb})$ tumor suppressor protein family members. CR3 contains two zinc finger motifs (4-8). E7 also contains a casein kinase II phosphorylation site that leads to the phosphorylation of E7 during the G1 and S phases of the cell cycle (5). One of the main functions of the E7 protein in the HPV life cycle is the binding and degradation of the $\mathrm{Rb}$ family of proteins. The $\mathrm{Rb}$ proteins are major regulators of the cell cycle. E7 protein is primarily localized in the nucleus, where it associates with the $\mathrm{pRb}$ to facilitate progression into the S-phase of the cell cycle. By associating with hypophosphorylated pRb, E7 prevents its binding with E2F, thereby promoting cell cycle progression. Besides pRb, E7 interacts with two other members of the pRb family, p107 and p130. This also negatively regulates E2F transcription $(7,9,10)$. 
In this study, we focused on surveying the genes and proteins modulated by E7 in the lung tissue of E7-transgenic mice. In addition, to elucidate whether E7 could activate putative biomarkers, such as cell cycle- and cancer cell adhesion- and migration-related genes in lung cancer development, we attempted to i) examine whether E7 modulates factors related to lung tumors; and ii) understand whether E7 oncogene expression in lung tissue was associated with the signaling pathway related to cancer development.

\section{Materials and methods}

Cell culture. Cervical cancer cell line CaSki containing 60-600 copies of the HPV-16 genome (11) was maintained in Dulbecco's modified Eagle's medium (DMEM) supplemented with $100 \mathrm{U} / \mathrm{ml}$ penicillin, $100 \mu \mathrm{g} / \mathrm{ml}$ streptomycin, $25 \mathrm{ng} / \mathrm{ml}$ amphotericin B (Gibco BRL, Grand Island, NY) and 10\% fetal bovine serum (FBS) (Hyclone, South Logan, UT) at $37^{\circ} \mathrm{C}$ in a humidified incubator with $5 \% \mathrm{CO}_{2}$.

Plasmid construction. cDNA was synthesized by RT-PCR from total RNA isolated from CaSki cells. The primers for E7 synthesis were as follows; sense 5'-CG GAA TTC ATG CAT GGA GAT ACA CCT ACA-3' and antisense 5'-CG GAA TTC AGC CAT GGT AGA TTA TGG TTT-3'. The PCR product was cloned into the EcoRI site of the pCAGGS vector, and then used to establish the E7-Tg mouse model.

Preparation of pCAGGS/E7 and establishment of transgenic mice. For the HPV16 E7 transgenic mouse model, concentrated E7 cDNA was prepared. pCAGGS/E7 plasmid was prepared using the Qiagen MIDI-prep kit. E7 transgenic mice were generated by DNA microinjection of $20 \mu \mathrm{g}$ E7 DNA into the embryos of BDF1 mice as previously described (12). Experimental treatments were carried out according to the guidelines for animal experimentation at the Faculty of Laboratory Animal Research Center, Konkuk University. E7 expression was confirmed with the isolated genomic DNA of Tg-mouse using Super Taq Plus Pre-mix (RexGene BioTech PCR Systems, Korea) and specific primer sets (PCR product 300 bp): sense 5'-CGT GCT GGT TGT TGT GCT GTC T-3' and antisense 5'-CGG AAT TCA GCC ATG GTA GAT TAT GGT TT-3'. pCAGGS/E7 was used as a positive control and pCAGGS (6.5 kbp) as a negative control. GAPDH was used as an internal control.

Genomics analysis. Gene expression analysis was conducted on RNA samples from the lung tissue of E7-Tg mice (female, 3 months old). Total RNA was prepared from mouse liver using TRIzol reagent (Invitrogen). For the microarray experiment, $10 \mu \mathrm{g}$ of total liver RNAs from two mice was used for cRNA synthesis. RNAs were pooled because pilot experiments with Affymetrix chips at Pfizer indicated that the inter-animal variability in gene expression as well as variability between repeated hybridizations of the same pooled RNA sample were statistically insignificant (13). Labeling and hybridization were performed using the Affymetrix 430 2.0 Array Kit (Affymetrix, Santa Clara, CA) following the manufacturer's instructions. Fluorimetric data were processed by Affymetrix
GeneChip3.1 software, and the gene chips were globally scaled to all the probe sets with an identical target intensity value. Affymetrix software measures the expression level of a gene as an average difference value (Avg Diff) by comparing the intensity of hybridization of 20 sets of perfect match oligonucleotide probes to 20 sets of mismatch probes. Genes that had an Avg Diff value above the threshold of 100 and at least 2-fold Avg Diff values between wild-type and E7-Tg mice were selected.

Proteomics analysis. Proteins were loaded on 4-20\% TrisGlycine Gel (Invitrogen, Carlsbad, CA). The gel fragments were excised in 10 bands according to molecular weight and digested with sequencing grade modified trypsin (Promega,

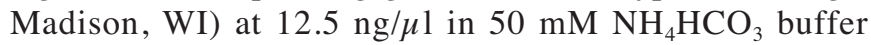
$(\mathrm{pH} 8.0)$ at $37^{\circ} \mathrm{C}$ overnight. The resulting tryptic peptides were loaded onto a fused silica microcapillary column $(15 \mathrm{~cm} \mathrm{x}$ $75 \mu \mathrm{m})$ packed with C18 (5 $\mu \mathrm{m}, 200 \AA$ ) reversed phase resin and were separated by liquid chromatography (LC) using a linear gradient of 5-50\% buffer A for $65 \mathrm{~min}$ followed by $50-90 \%$ buffer B for 5 min (buffer A, $0.1 \%$ formic acid in $\mathrm{H}_{2} \mathrm{O}$; buffer $\mathrm{B}, 0.1 \%$ formic acid in acetonitrile) at a flow rate of $250 \mathrm{nl} / \mathrm{min}$. The column was connected directly to LTQ iontrap mass spectrometry (Thermo Finnigan, Waltham, MA) equipped with a nano-electrospray ion source, and the eluent peptides were dynamically selected for fragmentation by the operating software. Protein identification was performed using Spectrum Mill Proteomics Workbench version A.03.03 (Agilent Technologies). Data files were extracted using the Spectrum Mill Data Extractor. Searches were carried out against the human NCBInr database in both forward and reverse directions using the Spectrum Mill program with the following parameters: specific to trypsin with two missed cleavage sites; $\pm 2.5 \mathrm{Da}$ precursor-ion tolerance; and $\pm 0.7 \mathrm{Da}$ fragment-ion tolerance. Carboxyamidomethylation of cysteine and oxidation of methionines were allowed as variable modifications. The initial results were auto-validated using the following parameters for details on the protein mode: scored percent intensity (SPI) $>70 \%$ for matches with a score $>7$ for $+1,>9$ for $+1,>9$ for $+3,>8$ for +4 ; and a SPI $>90 \%$ for a score $>6$ on +1 . The proteins were summarized by validated peptides using the following parameters: $>13$ for protein score; SPI $>70 \%$ for a score $>9$ on the peptide; and at least 2 distinct peptide hits included in each protein. A semi-quantitative analysis of protein profile data was performed by comparing total peptide intensity with the peptides of an identified protein. Total peptide intensity was obtained by summing up the peptide intensities of the peptide hits for the protein. False-positive rates were calculated as previously described (14).

RNA isolation and cDNA synthesis for Q-PCR analysis. Total RNAs were isolated from the lung tissue of E7 transgenic mice to measure the quantity of the various genes. The lungs of E7 transgenic and non-transgenic mice were collected, homogenized, and lysed using TRIzol. Total RNAs were cleaned up using the RNeasy Mini Kit (Qiagen, CA) according to the manufacturer's protocol. The total RNA ( $3 \mu \mathrm{g})$ of each mouse and $3 \mu \mathrm{g}$ of oligo(dT) were used for cDNA synthesis using reverse transcriptase (BioLabs). 
Table I. List of primers used for Q-PCR.

\begin{tabular}{lll}
\hline Gene & \multicolumn{1}{c}{ Forward } & Reverse \\
\hline GAPDH & ACCACAGTCCATGCCATCAC & TCCACCACCCTGTTGCTGTA \\
Cyclin B1 & GGGTCACTAGGAACACGAAAA & CCAATGTCTCCAAGAGCAGTT \\
Cyclin E2 & ATGTCAAGACGCAGCCGTTTA & TGATGCTTCTTGGTGATCTCC \\
Activated leukocyte cell adhesion molecule (CD166) & ATGGCATCTAAGGTGTCCCCT & AGACGGCAAGGCATGACAA \\
Actin $\alpha 1$ & AACGACTACATGCAGCCTGAA & GTCCTCTTCGATGTTCTCGA \\
Calnexin & TGGTTACTGTGTTTGCTGCTG & AGGAGTGCTGGCATCTGATTT \\
Destrin & AGTTCAGGTTGCGGATGAAGT & TGCACTTTTTGTCTGCACTG \\
Protein disulfide isomerase-associated 3 & ATGCGCTTCAGCTGCCTA & AGAACTCGATAGCATGAGCC \\
Vimentin & ATTTCTCTGCCTCTGCCAAC & CCTGTCCATCTCTGGTCTCAA \\
Cofilin 1 & GACGACCCCTACACCACTTTT & TCCTCCTTCTTGCTCTCCTT \\
Cdc 46 & TGGTGGGGTTGTCTGTATTG & GAGCAGCGAGAGTTCAAGGT \\
Gelsolin & TATCAGRGGRGRGGCTCTGG & CTTCTGGCTCTCCTCCCTCT \\
\hline
\end{tabular}

Real-time quantitative PCR. Gene expression levels of the E7-Tg mice were confirmed using Bio-Rad SYBR Green quantitative real-time PCR (Bio-Rad, CA). Primer pairs producing a unique band and no primer dimerization were selected for real-time PCR assays. The sequences of the PCR primer pairs are listed in Table I. Data were analyzed according to the manufacturer's instructions. The quantitative values for gene expression were obtained by relative comparison with GAPDH expression level. All values were calculated from three independent trials. The threshold cycle (CT) indicates the fractional number at which the amount of amplified target reaches a fixed threshold. ${ }^{\wedge} \mathrm{CT}=$ target gene $(\mathrm{CT})$ - control gene (housekeeping gene) $(\mathrm{CT}) .{ }^{\Delta} \mathrm{CT}=$ target gene $\left({ }^{\Delta} \mathrm{CT}\right)-$ control gene $\left({ }^{\wedge} \mathrm{CT}\right)$. Fold difference $=2^{-\Delta \Delta C T}$.

\section{Results}

Establishment and confirmation of E7 transgenic mice. We attempted to verify that E7 cDNA was properly translated into E7 protein before injecting it into the mice. E7 was fused with GST and then expressed in Escherichia coli. GST-fused E7 protein was detected in Western blotting using the antibody to $\mathrm{E} 7$ at $\sim 40 \mathrm{kDa}$ (Fig. 1A). E7 cDNA was cloned into mammalian expression vector pCAGGS and then used for E7 transgenic mouse production. E7 transgenic mice were confirmed by RT-PCR with genomic DNA extracted from their tail pieces. PCR product of $300 \mathrm{bp}$ was observed on $1 \%$ agarose gel (Fig. 1B), suggesting that four transgenic mice were produced at this mating.

Histopathological examination and selection of E7-Tg mice for the identification of E7 modulators. E7 male mice aged 3 and 7 months were sacrificed. Lungs were removed and compared with lungs from non-Tg mice. There was no significant phenotypic difference between the two groups (data not shown). It has been reported that $\sim 10 \%$ of $\mathrm{E} 7$ transgenic mice carrying two copies of the K14-HPV16, which is expressed in squamous epithelia, spontaneously develop skin tumors at 10 months of age as well as cervical tumors (with estrogen treatment) (15). On the basis of this report, we used 3-month-
A

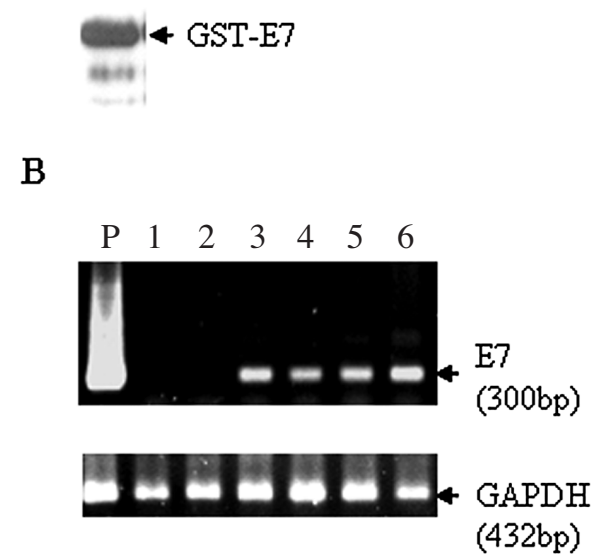

Figure 1. Western blot analysis of GST-fused E7 recombinant protein and screening of E7 transgenic mice. (A) GST-E7 was purified from E. coli, resolved by SDS-PAGE, transferred onto a nitrocellulose membrane, and then detected with anti-E7 antibody. (B) Screening of E7 transgenic mice. Genomic DNA was isolated from the tails of the mice, and E7-expressing mice were screened by PCR. pCAGGS/E7 was used as a positive control (lane P) and pCAGGS as a negative control (lane 1). Lane 2, a non-Tg mouse.

old mice for omics analysis to identify biomarkers related to HPV and epithelial cancer at an early developmental stage.

Gene expression profiles of the lung tissues of E7-Tg mice. As shown in Fig. 2 and Table II, we respectively categorized up- and down-regulated genes according to their biological functions. Genes related to metabolism, cellular and/or physiological processes and muscle fiber development were the most modulated in their expression levels. Among the upregulated genes, the proportion of genes related to cell cycle and macromolecule metabolism was high, and that of several genes involved in nucleotide metabolism was moderate. Genes related to cell organization and biogenesis, muscle fiber development and response to stress were prone to be decreased. Genomics analysis using E7-Tg mice showed that sarcolipin, titin, cyt p450 and proteoglycan 4 were down- 
Table II. Genes regulated by the E7 oncogene.

A. Genes up-regulated in lung tissue.

\section{Gene}

Cyclin E2

Centrosomal protein 55

Helicase, lymphoid specific

Cyclin B1, related sequence 1/predicted gene, EG434175/similar to G2/mitotic-specific cyclin-B1

Growth arrest-specific 2 like 3

PDZ binding kinase

Ribonucleotide reductase M2

Transcription factor 19

Antigen identified by monoclonal antibody Ki 67/similar to antigen KI-67

Calcitonin/calcitonin-related polypeptide, $\alpha$

Cell division cycle 20 homolog (S. Cerevisiae)

Minichromosome maintenance deficient 5, cell division cycle 46

Chromatin licensing and DNA replication factor 1

Ribonucleotide reductase M2

Checkpoint kinase 1 homolog (S. Pombe)

Centromere protein $\mathrm{F}$

Cell division cycle 2 homolog A (S. Pombe)

Topoisomerase (DNA) II $\alpha$

Transformation related protein 53 inducible protein 5

Similar to histone $2 \mathrm{a}$

Replication factor C (activator 1) 4

Rac gtpase-activating protein 1

Cell division cycle 20 homolog (S. Cerevisiae)

Chitinase 3-like 4

Ubiquitin-like, containing PHD and RING finger domains, 1 $\log 2$ ratio

3.200

3.192

3.145

3.004

2.968

2.946

2.912

2.893

2.813

2.675

2.581

2.562

2.537

2.535

2.523

2.519

2.504

2.475

2.473

2.459

2.447

2.441

2.416

2.405

B. Genes down-regulated in lung tissue.

Gene

$\log 2$ ratio

Creatine kinase, mitochondrial 2

Myosin, heavy polypeptide 6 , cardiac muscle, $\alpha$

$-1.572$

Proteoglycan 4 (megakaryocyte stimulating factor, articular superficial zone protein)

$-1.566$

Cytochrome P450, family 1, subfamily a, polypeptide 1

$-1.476$

Serum amyloid A 3

$-1.460$

Myosin, light polypeptide 7, regulatory

$-1.446$

Sarcolipin

$-1.440$

Titin

$-1.424$

Myoglobin

$-1.389$

Troponin I, cardiac

Hepcidin antimicrobial peptide 1

Titin-cap

Myosin, heavy polypeptide 6 , cardiac muscle, $\alpha /$ myosin, heavy polypeptide 7 , cardiac muscle, $B$

Neurotrimin

Solute carrier family $15\left(\mathrm{H}^{+} /\right.$peptide transporter $)$, member 2

Taxilin $B$

Myosin, light polypeptide 4

$-1.178$

Transforming growth factor, $B$ receptor III

$-1.170$

Actinin $\alpha 2$

$-1.166$

Heat shock protein 1B

$-1.165$

Troponin T2, cardiac

Polycystic kidney disease 1 like 2

Potassium inwardly-rectifying channel, subfamily J, member 3 

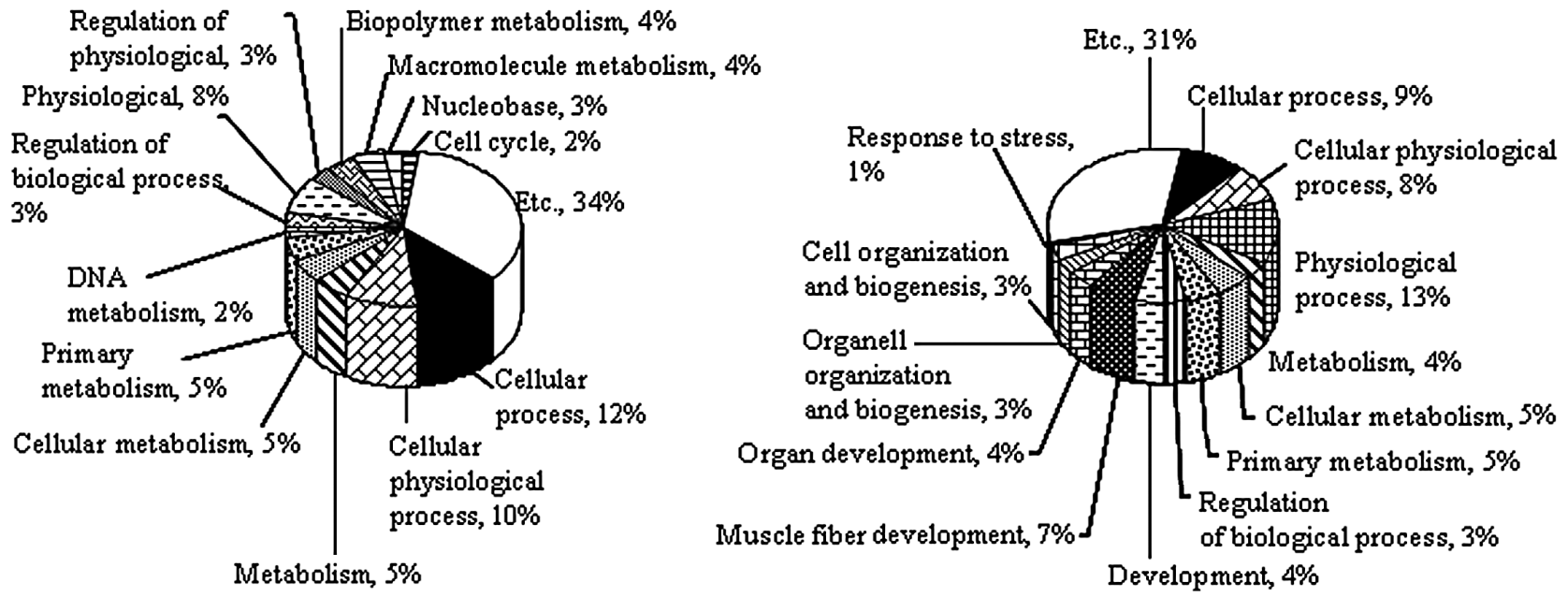

Figure 2. Pie charts showing the distributions of (A) up-regulated and (B) down-regulated genes based on their biological function in the lung tissue of E7 transgenic mice.

Table III. Proteomics analysis of protein profiling of lung proteins in the E7-Tg mice.

\begin{tabular}{lcc}
\hline \multirow{2}{*}{ Fold change } & \multicolumn{2}{c}{ Mus musculus DB } \\
\cline { 2 - 3 } & Up-regulated & Down-regulated \\
\hline$<3$ & 21 & 27 \\
$3-5$ & 12 & 15 \\
$5-9$ & 10 & 25 \\
$>10$ & 93 & 150 \\
Total & 136 & 217 \\
\hline
\end{tabular}

Data files were extracted using the Spectrum Mill Data Extractor with the parameters of $\left[\mathrm{MH}^{+}\right] 600-40000$ and minimum signal-to-noise (S/N) 25. Searches were carried out against the human NCBInr database in both forward and reverse directions using the Spectrum Mill program (Agilent Technologies) with the following parameters: specific to trypsin with two missed cleavage sites; \pm 2.5 Da precursorion tolerance; and $\pm 0.7 \mathrm{Da}$ fragment-ion tolerance. The initial results were auto-validated as described in Materials and methods. Acceptance scores: protein $>13$; peptide $>9$; score peak intensity $>70 \%$. DB, NCBI database.

regulated, while topoisomerase II $\alpha$ as well as cell cycle-related genes such as centrosomal protein 55, cylin B1, cyclin E2, checkpoint kinase 1 and cell division cycle 46 were upregulated by the E7 oncogene in the lung tissues of $\mathrm{Tg}$ mice (Table II)

Protein expression profiles of the lung tissue of E7-Tg mice. Proteins modulated by the E7 oncogene were analyzed by liquid chromatography/electrospray ionization tandem mass spectroscopy (LC-ESI-MS/MS). In the case of E7-Tg mice, 136 up-regulated proteins and 217 down-regulated proteins were classified by fold change, as shown in Table III. Proteins up- and down-regulated by the E7 oncogene were classified according to the Gene Ontology database (http://www . geneontology.org/) based on cellular composition and the biological process using the house-made FindGo program as shown in Fig. 3. After a comparison of the protein expression profiles of normal and E7-Tg mouse lungs, we constructed pie charts that divided several types of proteins based on their cellular composition (Fig. 3A). Upon analysis of the upregulated proteins, cell proliferation-related and nuclear and intracellular proteins were most apparent in E7-Tg mice, while proteins related to the protein complex as well as mitochondrial and extracellular proteins were principally observed in normal mice. Golgi apparatus-, endoplasmic reticulum- and cytoskeleton-related proteins showed no variation between normal and E7-Tg mice. We also classified proteins with known functions based on their involvement in biological processes, as shown in Fig. 3B. It was found that proteins involved in translation/transcription nucleotide sequences $(5$ to $>6 \%$ ) as well as transport/localization (16 to $>18 \%$ ) were increased, while those involved in protein folding ( 2 to $>1 \%$ ) and cell adhesion ( 3 to $>2 \%$ ) were decreased in the lung adenomas of E7-Tg mice. Significantly up- and downregulated proteins found by comparing the protein expression profiles of normal and E7-Tg mouse lungs are listed in Table IV. Glutathione S-transferase, cofilin, destrin, Rho GDP dissociation inhibitor $\alpha$, ADP-ribosylation factor 2, vimentin, drep1 protein, and protein disulfide isomerase associated 3 were down-regulated, while gelsolin, $\mathrm{Na}^{+} / \mathrm{K}^{+}$-ATPase $\alpha 1$ subunit, UDP-glucose ceramide glucosyl transferase, mitochondrial malate dehydrogenase, calnexin, platelet glycoprotein, activated leukocyte cell adhesion molecule CD166, diaphorase, actinin $\alpha 1$, and annexin A2 and A4 were up-regulated by the E7 oncogene in the lung tissue of transgenic mice (Table IV).

Evaluation of E7-modulators by quantitative real-time PCR. Quantitative real-time PCR analysis was used to measure the quantity of transcripts modulated by the E7 oncogene in the 


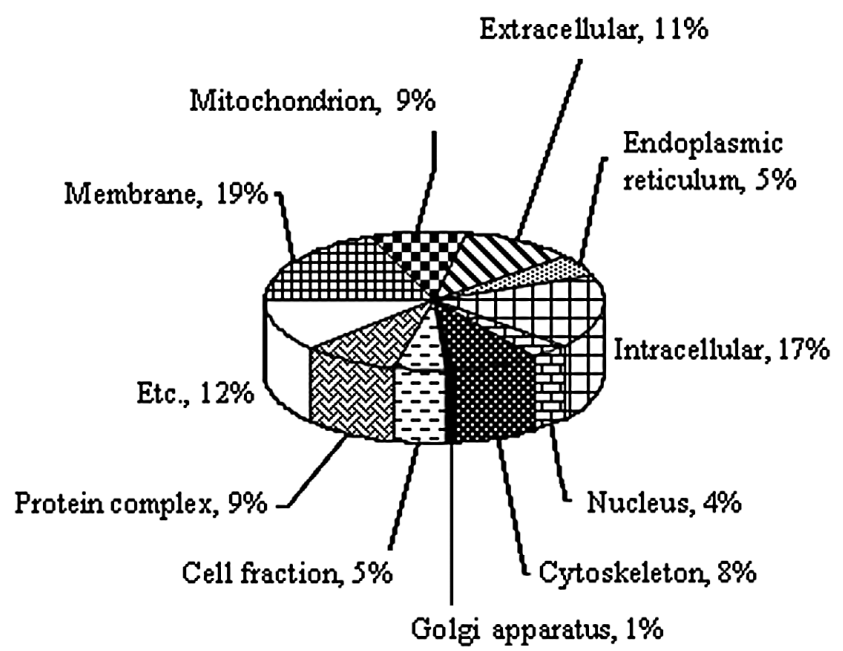

B

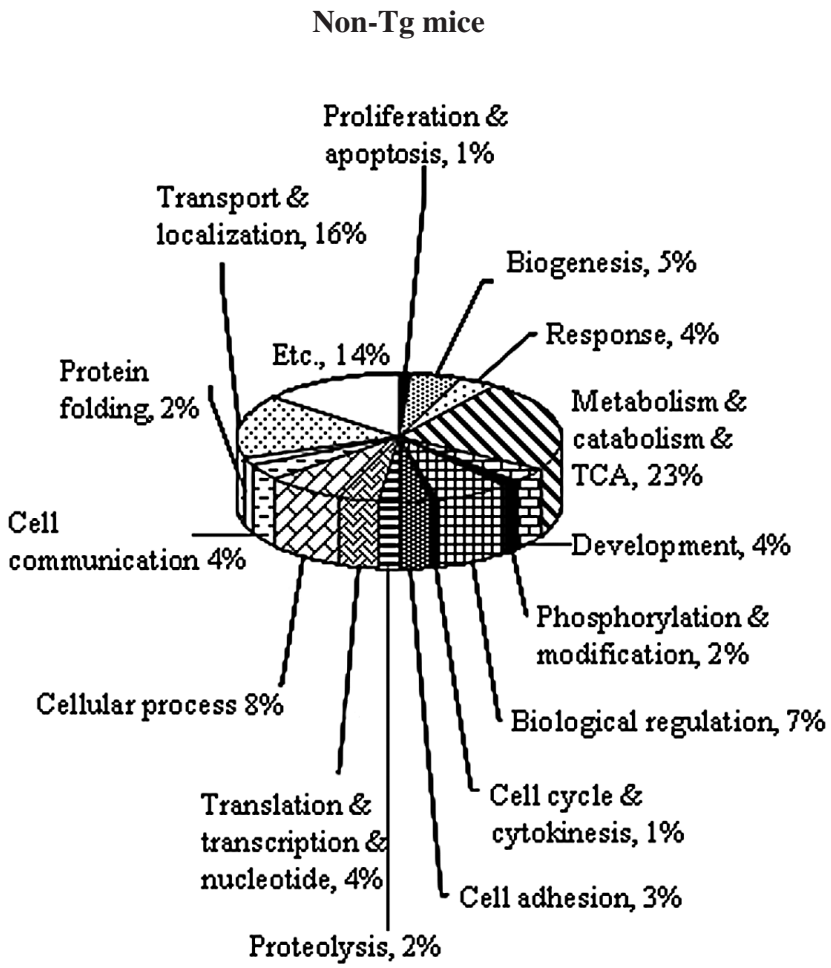

E7-Tg mice

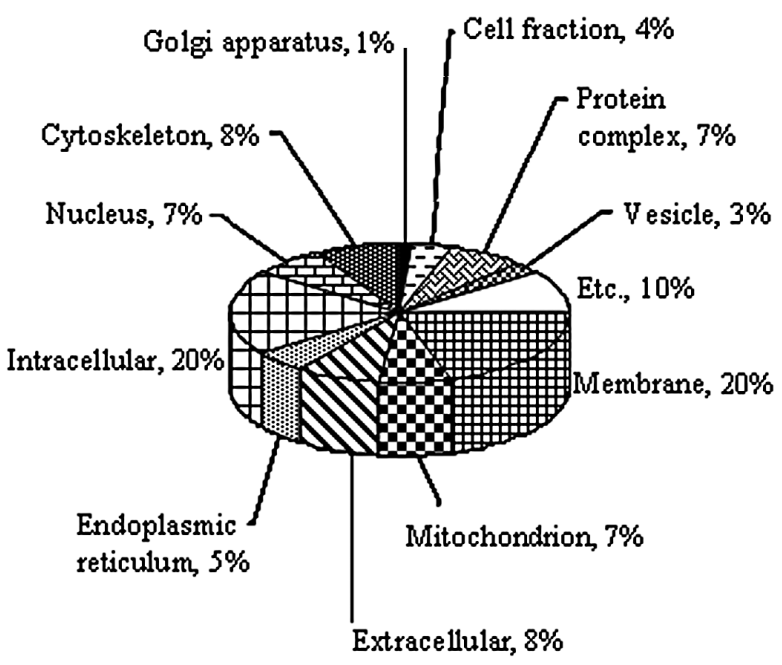

E7-Tg mice

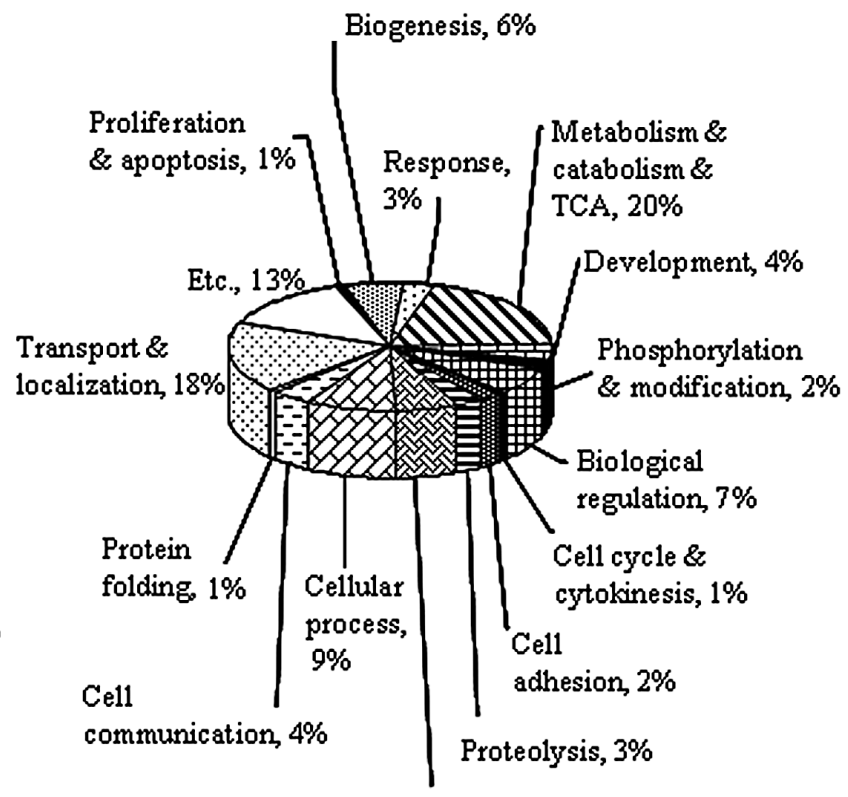

Translation \& transcription nucleotide, $6 \%$

Figure 3. Pie charts showing protein categorization in the lung tissue of non-Tg and E7-Tg mice based on (A) cellular composition and (B) biological process. Proteins up- and down-regulated by the E7 oncogene were classified into categories according to the gene ontology database based on cellular composition and the biological process using the house-made FindGo program.

lung tissues of E7-Tg mice. Non-TG mice were used as the control in this experiment. Real-time PCR was performed for the selected genes related to cancer cell adhesion, cell cycle and migration, and for the genes related to proliferation and apoptosis listed in Tables II and IV. The gene and protein expression data obtained from omics analysis were consistent with the real-time PCR analyses (Fig. 4). The results indicated that cyclin B1, cyclin E2, activated leukocyte cell adhesion molecule CD166, actinin $\alpha 1$, calnexin and gelsolin were upregulated, while cofolin-1 and vimentin were down-regulated by the E7 oncogene (Fig. 4).

\section{Discussion}

Viral oncogenes such as HPV16 E6, HPV16 E7, HBV X and adenovirus EIA have been known to bind to p53 and Rb, which are typically anticancer genes. As a consequence of the blocking of the functions of these antitumor genes, cancer is induced. In the case of the E7-Tg mouse model, although it was developed and distributed by The National Cancer Institute/ Mouse Models for Human Cancer Consortium (Bethesda, MD, USA), the implantation percentage of the fertilized eggs was too low and the incidence of malignant tumors was less than 
Table IV. Proteins regulated by the E7 oncogene.

A. Up-regulated proteins in lung tissues.

Entry name

Unnamed protein product

Gelsolin

Mkiaa 0034 protein

Mitochondrial trifunctional protein, $\alpha$ subunit

Activated leukocyte cell adhesion molecule CD166

$\mathrm{Na}^{+} / \mathrm{K}^{+}$-atpase $\alpha 1$ subunit

Actinin, $\alpha 1$

Calnexin

Lactate dehydrogenase 2, B chain

Annexin A2

Heat shock protein $1, \beta$

Similar to spectrin $\alpha$ chain, brain isoform 18

Angiotensin I converting enzyme (peptidyl-dipeptidase A) 1 isoform 1

Solute carrier family 25 , member 5

Xanthine dehydrogenase

Platelet glycoprotein 4

Brain $\beta$ spectrin

Guanine nucleotide binding protein $\beta 4$

Diaphorase 1

Mkiaa0051 protein

UDP-glucose ceramide glucosyltransferase-like 1

Nuclear myosin IB

Annexin A4

Vacuolar protein sorting 35

Laminin, $\gamma 1$

Malate dehydrogenase 2, NAD (mitochondrial)
E7-Tg/non-Tg intensity

B. Down-regulated proteins in lung tissues.

Entry name

Unnamed protein product

Serpina1c protein

Glutathione S-transferase, mu 2

Destrin

PREDICTED: similar to peptidyl-prolyl cis-trans isomerase A

ADP-ribosylation factor 2

PREDICTED: similar to PRAME family member 9

Protein disulfide isomerase associated 3

PREDICTED: similar to transgelin 2

mKIAA 0002 protein

PREDICTED: similar to myosin light polypeptide 6

Advanced glycosylation end product-specific receptor

Flavin-containing monooxygenase 2

PREDICTED: similar to superoxide dismutase isoform 4

Isocitrate dehydrogenase [NADP], mitochondrial precursor

Hemopexin

Heat shock protein 9A

Dihydropyrimidinase-like 2

PREDICTED: similar to aspartate aminotransferase, mitochondrial

Cofilin 1, non-muscle

Rho GDP dissociation inhibitor (GDI) $\alpha$

Moesin

Rps16 protein

Atp5b protein

Vimentin
Non- $\mathrm{Tg} / \mathrm{E} 7 \mathrm{Tg}$ intensity

240.472

98.750

52.217

38.511

33.106

32.733

31.030

27.566

27.325

24.566

23.325

18.131

17.666

16.216

15.185

14.608

14.378

13.846

12.792

11.589

10.556

9.192

8.596

8.250

4.491 

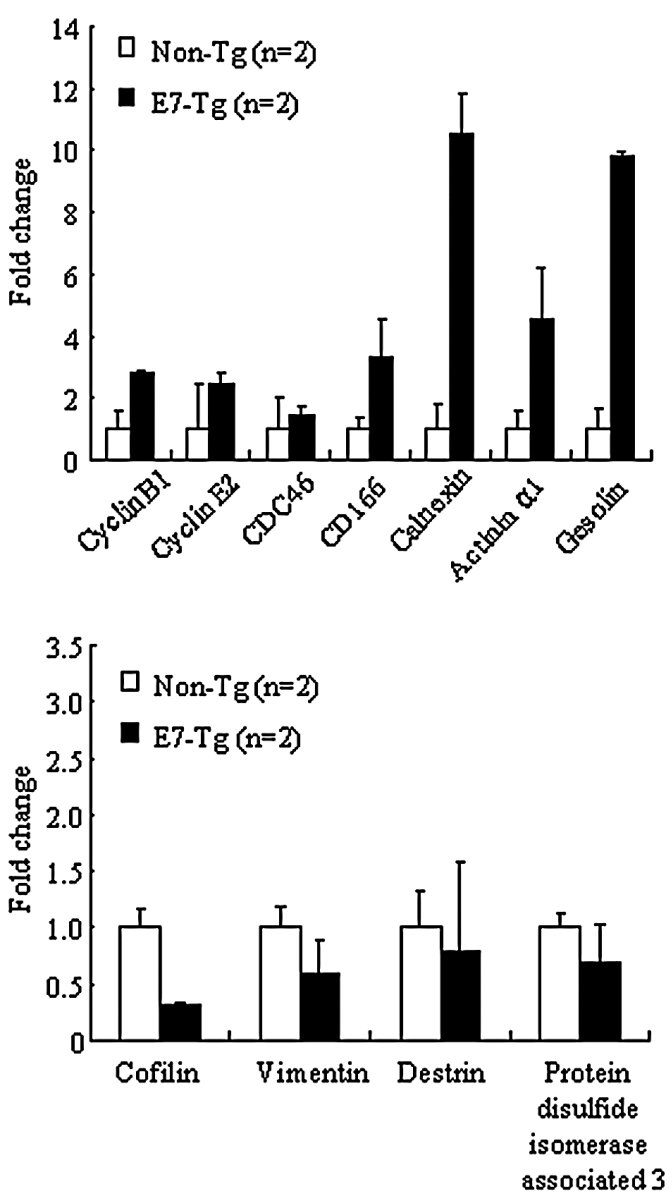

Figure 4. Real-time PCR was performed analyzing the candidates of significance from genomics and proteomics data to measure expression levels. The respective fold changes were expressed by comparison with the values of non- $\mathrm{Tg}$ mice which were set as 1 . Mean values of triplicates of each gene were compared.

$10 \%$, so distribution has currently been halted. As a result, the transgenic mice expressing HPV16 E7 oncoprotein used in the present study were constructed by microinjecting the E7 recombinant gene into normal mice. Two transgenic mice (nos. 153 and 155) were confirmed to be transformed with the E7 oncogene by PCR. Mouse no. 155 was bred to generate ten littermates, and four transgenic mice were finally identified out of the ten (Fig. 1B). The lungs of 3- and 7-month-old E7 male mice were removed and compared with those of non-Tg mice, revealing that there was no significant difference between the two groups (data not shown). It has previously been demonstrated that ectopic expression of HPV-16 E6 and E7 in the skin of one HPV-16 transgenic mouse lineage can induce squamous cell carcinomas in $25 \%$ of the adult mice (16). However, Greenhalgh et al also directed expression of HPV-16 E6 and E7 together to the epidermis using a suprabasal layer-specific K1 promoter. Their mice also exhibited epidermal hyperplasia and, at lower frequencies, the incidence of papillomas (17). Arbeit et al directed expression of the entire early region of HPV-16 to the epidermis by using the same K14 promoter, but failed to detect squamous cell carcinomas (18). In these studies, 3-month-old E7 transgenic mice were used for omics analysis in order to identify transcripts and proteins related to HPV and epithelial cancer at the early developmental stage.
Here, omics data demonstrated that the E7 oncogene up-regulated genes related to the cell cycle and adhesion and migration, which may lead to oncogenesis. Two intermediate filament proteins, vimentin and cofilin-1, were downregulated, while gelsolin was up-regulated by the E7 oncogene (Fig. 4). Destrin was not significantly down-regulated in the lung tissues of E7-Tg mice. Cofilin-1 and destrin promote cytoskeletal dynamics by depolymerizing actin filaments. Cells that knocked-down those genes also had defects in cell motility and cytokinesis, caused by diminished actin filament depolymerization rates (19). It is well known that apoptotic cells are significantly reduced by levels of the intermediate filament proteins, keratins-18, -19 , vimentin and the associated 14-3-3 adapter proteins (20). Calnexin was markedly increased while protein disulfide isomerase associated 3 was not significantly altered in the lung tissues of E7-Tg mice (Table IV). In vivo, it is likely that cytoplasmic actin binds and inhibits the enzymatic activity and nuclear translocation of DNase I, and that disruption of the actin-DNase I complex results in activation of DNase I. It was demonstrated that the N-terminal fragment of gelsolin (N-gelsolin) disrupts actin-DNase I interaction, and that cofilin stabilizes the actin-DNase I complex by forming a ternary complex that prevents $\mathrm{N}$-gelsolin from releasing DNase I from actin, suggesting that both cofilin and gelsolin are essential in modulating the release of DNase I from actin (21). Collectively, previous and our present data indicate that apoptosis-associated processes in lung epithelial cells include solubilization of the rigid intermediate filament network by specific proteolysis as well as increased levels of endoplasmic reticulum proteins with chaperone functions (20). It is presumed that small interfering RNA-mediated silencing of actinin- $1 \alpha$ in tumor tissue can disrupt E7-stimulated cancer cell adhesion and migration and thereby inhibit subsequent cancer cell growth. In these studies, we identified E7-modulated biomarkers such as cell cycle- (cyclin B1, cyclin E2) and cancer cell adhesion- and migration- (CD166, actinin $\alpha 1$ ) related genes, which may play important roles in cellular transformation in cancer. In addition, solubilization of the rigid intermediate filament network by specific proteolysis mediated via up-regulating gelsolin and down-regulating cofilin-1, as well as increased levels of endoplasmic reticulum proteins with chaperone functions, might also be involved in E7-lung epithelial cells.

\section{Acknowledgements}

This work was supported by omics program 08162-510 from the Korea Food and Drug Administration and in part by KOSEF (grant R01-2006-101450) and the Korea Research Foundation Grant 2006-E00119.

\section{References}

1. Munoz N, Bosch FX, De Sanjose S, et al: Epidemiologic classification of human papillomavirus types associated with cervical cancer. N Eng J Med 348: 518-527, 2003.

2. Phelps WC, Yee CL, Munger K and Howley PM: The human papillomavirus type $16 \mathrm{E} 7$ gene encodes transactivation and transformation functions similar to those of adenovirus E1A. Cell 53: 539-547, 1988.

3. Boyer SN, Wazer DE and Band V: E7 protein of human papilloma virus-16 induces degradation of retinoblastoma protein through the ubiquitin-proteasome pathway. Cancer Res 56: 4620-4624, 1996. 
4. Clemens KE, Brent R, Gyuris J and Munger K: Dimerization of the human papillomavirus E7 oncoprotein in vivo. Virology 214: 289-293, 1995

5. Alva JA, Zovein AC, Monvoisin A, et al: VE-Cadherin-Crerecombinase transgenic mouse: a tool for lineage analysis and gene deletion in endothelial cells. Dev Dyn 235: 759-767, 2006.

6. Dahiya A, Gavin MR, Luo RX and Dean DC: Role of the LXCXE binding site in Rb function. Mol Cell Biol 20: 6799-6805, 2000.

7. Clements A, Johnston K, Mazzarelli JM, Ricciardi RP and Marmorstein R: Oligomerization properties of the viral oncoproteins adenovirus E1A and human papillomavirus E7 and their complexes with the retinoblastoma protein. Biochemistry 39 : 16033-16045, 2000

8. Dyson N, Howley PM, Munger K and Harlow E: The human papilloma virus-16 E7 oncoprotein is able to bind to the retinoblastoma gene product. Science 243: 934-937, 1989.

9. Alonso LG, Garcia-Alai MM, Smal C, et al: The HPV16 E7 viral oncoprotein self-assembles into defined spherical oligomers. Biochemistry 43: 3310-3317, 2004.

10. Phelps WC, Munger K, Yee CL, Barnes JA and Howley PM: Structure-function analysis of the human papillomavirus type 16 E7 oncoprotein. J Virol 66: 2418-2427, 1992.

11. Le Naour F, Hohenkirk L, Grolleau A, et al: Profiling changes in gene expression during differentiation and maturation of monocytederived dendritic cells using both oligonucleotide microarrays and proteomics. J Biol Chem 276: 17920-17931, 2001.

12. An J, Yuan Q, Wang C, et al: Differential display of proteins involved in the neural differentiation of mouse embryonic carcinoma P19 cells by comparative proteomic analysis. Proteomics 5: 1656-1668, 2005.

13. Zandbergen F, Mandard S, Escher P, et al: The G0/G1 switch gene 2 is a novel PPAR target gene. Biochem J 392: 313-324, 2005.
14. Qian WJ, Liu T, Monroe ME, et al: Probability-based evaluation of peptide and protein identifications from tandem mass spectrometry and SEQUEST analysis: the human proteome. J Proteome Res 4: 53-62, 2005

15. Riley RR, Duensing S, Brake T, Munger K, Lambert PF and Arbeit JM: Dissection of human papillomavirus E6 and E7 function in transgenic mouse models of cervical carcinogenesis. Cancer Res 63: 4862-4871, 2003

16. Lambert PF, Pan H, Pitot HC, Liem A, Jackson M and Griep AE: Epidermal cancer associated with expression of human papillomavirus type $16 \mathrm{E} 6$ and E7 oncogenes in the skin of transgenic mice. Proc Natl Acad Sci USA 90: 5583-5587, 1993.

17. Greenhalgh DA, Wang XJ, Rothnagel JA, et al: Transgenic mice expressing targeted HPV-18 E6 and E7 oncogenes in the epidermis develop verrucous lesions and spontaneous, rasHaactivated papillomas. Cell Growth Differ 5: 667-675, 1994.

18. Arbeit JM, Munger K, Howley PM and Hanahan D: Progressive squamous epithelial neoplasia in K14-human papillomavirus type 16 transgenic mice. J Virol 68: 4358-4368, 1994.

19. Hotulainen P, Paunola E, Vartiainen MK and Lappalainen P: Actin-depolymerizing factor and cofilin-1 play overlapping roles in promoting rapid $\mathrm{F}$-actin depolymerization in mammalian nonmuscle cells. Mol Biol Cell 16: 649-664, 2005.

20. Prasad SC, Soldatenkov VA, Kuettel MR, Thraves PJ, Zou X and Dritschilo A: Protein changes associated with ionizing radiation-induced apoptosis in human prostate epithelial tumor cells. Electrophoresis 20: 1065-1074, 1999.

21. Chhabra D, Nosworthy NJ and Dos Remedios CG: The $\mathrm{N}$-terminal fragment of gelsolin inhibits the interaction of DNase I with isolated actin, but not with the cofilin-actin complex. Proteomics 5: 3131-3136, 2005. 
